Journal of Electronics Voltage and Application

\title{
Smart Fish Feeder Using Solar Energy
}

\author{
Mohammad Danial Shahiran $^{1}$, Suriana Salimin ${ }^{1 *}$ \\ ${ }^{1}$ Green and Sustainable Energy Focus Group (GSEnergy), Faculty of Electrical and Electronic Engineering \\ Universiti Tun Hussein Onn Malaysia, 86400 Parit Raja, Batu Pahat, Johor, MALAYSIA \\ *Corresponding Author
}

DOI: https://doi.org/10.30880/jeva.2021.02.02.010

Received 04 July 2021; Accepted 29 September 2021; Available online 30 December 2021

\begin{abstract}
Smart fish feeder is an emerging concept of the current trend which use Internet of Things (IoT) technology to operate, monitoring and provides crucial information and status to the whole farming system. This project aims to provide such essential proof of concept that utilized IoT technology combine with the solar energy to power up servo motor and temperature sensor that connect from NodeMCU for the agriculture system. The main objectives of this project are specifically focused on the development of a smart fish feeder by using the solar system with charging capability and controlled by the IoT system. Such a fish feeder system was powered up by $12 \mathrm{~V}$ battery using $10 \mathrm{~W}$ solar panel controlled by a solar charger controller. The solar energy was stored in $12 \mathrm{~V}$ rechargeable battery. IoT-controlled sensors were also attached to the fish feeder system for providing essential information on temperature and fish feeder timer via the Blynk platform. The results of the developed system successfully proved the concept is workable and could be extended to a larger scale of the farming industry. Owing to its energy autonomy and low cost, the system has the potential to be useful in smart farming technology.
\end{abstract}

Keywords: Fish Feeder, Blynk App, IoT

\section{Introduction}

In the last few years, renewable energy systems have piqued the public's interest due to the limited availability of conventional energy sources and the numerous issues that come with their use, such as pollution and high grid demands [1]. Renewable energy often denoted as clean energy comes from natural resources or processes that are constantly replenished. Solar energy is preferred among renewable energy sources because it has the potential to provide the cleanest, most sustainable energy for the next few billion years [2]. It has not harmed the environment or people themselves. As the world is more dependent on technology, there is greater dependence on finding more renewable energy to sustain this technology.

Agriculture has increased every year according to the Department of Statistics 2019. Crops, livestock, and fisheries are the three main agriculture sub-sectors covered in these statistics. In 2018, the agriculture sector contributed 7.3 percent of the Gross Domestic Product (GDP), or RM99.5 billion. In comparison to the other sectors, the fisheries sector only contributes 12.5 percent to GDP. The total marine fish landings in 2018 were 1,476.9 thousand tonnes, up 0.8 percent from the previous year's 1,465.1 thousand tonnes. Malaysian freshwater aquaculture production also increased by 3.1 percent. [3].

In the aquatic system, freshwater fish are the major and the most occupied in the topic cascade [4]. The increasing amount in the statistics means it increases the manpower to feed and monitor the quality of water in the pond. Any deviation from the allowed parameter range can result in an undesirable scenario such as disease, stress, death, and profit loss, so quality must be continuously monitored [5-8]. Different types of fish and aquatic life require different levels of water quality, including temperature, hydrogen potential $(\mathrm{pH})$, and other factors. As a result, critical parameters such as 
water temperature must be continuously monitored to ensure the production of healthy fish [9]. The majority of fishpond farmers in Malaysia currently monitor the water temperature manually using a portable sensor [10].

However, the main problem in this manual method is they need to check the water temperature one by one of the fishponds. The other problem that occurs is the supply of electrical energy that requires to turn on the servo motor to feed the fish. For the time being most of fishpond farmers use direct electrical energy to turn on the fish feeder system. But, if the blackout happens at the farm the system will be disturbing and the system may be failed due to blackout happen. Solar energy is needed to back up the fish feeder system to make sure there is no miscellaneous problem occur. Maintaining a good quality of water is important for the fishpond farmers. In order to improve the manual method, the development of a Smart Fish Feeder Using Solar Energy is crucial to be embedded to eliminate the manual feeding and manual water quality monitoring. Thus, this research aims to design and develop a Smart Fish Feeder Using Solar Energy that is capable of automatically monitoring and recording water temperature. The industry will benefit from the implementation of the developed system in terms of manpower and time management. The developed system will be capable of not only continuously monitoring the parameter but also recording and analysing each reading in a more efficient manner.

\section{Project Methodology}

\subsection{Project System Design}

The hardware and software for this project are being developed. The development of a project is usually divided into two parts. The first section focuses on hardware development and design, while the second section focuses on software development. In order to develop the hardware of the smart fish feeder system, there are several components that are needed. The components installed in the smart fish feeder system consist of NodeMCU, DS18B20 sensors and Servo Motor. Software is used to monitor and control the servo motor. In the part of the software, NodeMCU is needed to install the program code. The program code is written for the smart fish feeder system. After both the hardware and software are built, the hardware will be connected with the software of the mobile phone. Figure 1 shows the flowchart of the designing and development of hardware and software. As in Fig. 1 below, the first step is to design the Smart Fish Feeder that is suitable for all the component that is used by Sketch Up. After that, the coding will be created by using Arduino IDE. The crucial part of this project is to determine PV System Sizing by using a formula that can support the whole system in the Smart Fish Feeder. When all the steps have been taken the Smart Fish Feeder will be developed. Next, the parameter for the servo motor will be set using Arduino IDE with the right angle to make sure the pellet will disperse in the correct amount. Lastly, the output of pellet disperses and water temperature is fully functional using Blynk Apps.

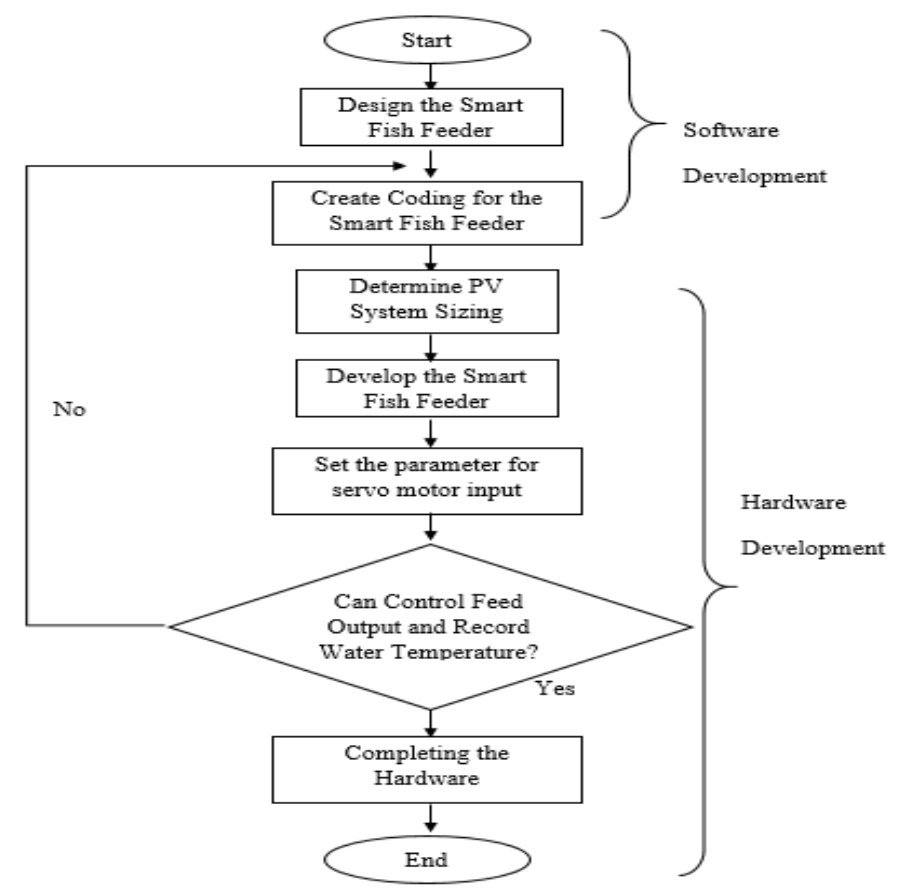

Fig. 1 - Detail flowchart on hardware and software development 


\subsection{Hardware Component}

The development of the transmitter unit employed NodeMCU which connected with the servo motor and temperature sensor. The NodeMCU act as the processing unit to control the servo motor within the timer and DS1820 to measure the temperature. On the other hand, the solar charger controller is connected between the solar panel and battery. Fig. 2 shows the circuit diagram of the Smart Fish Feeder Using Solar Energy.

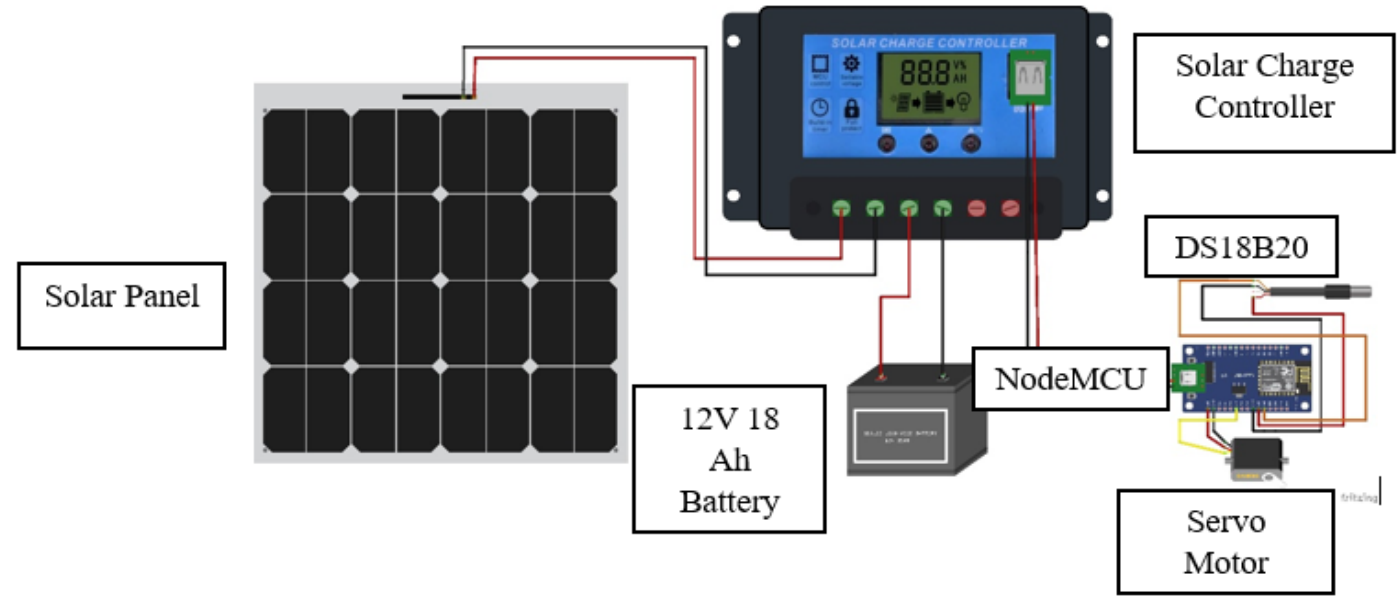

Fig. 2 - Smart Fish Feeder using solar energy circuit diagram

\subsection{Prototype Load Calculation}

Table 1 shows the watt rating of loads and the result of the energy used per day based on the duration of the operation.

Table 1 - Calculation of component rating

\begin{tabular}{ccccc}
\hline Item & Watts & Quantity & $\begin{array}{c}\text { Duration } \\
\text { (Hours) }\end{array}$ & $\begin{array}{c}\text { Total Wh } \\
\text { per day }\end{array}$ \\
\hline $\begin{array}{c}\text { NodeMCU } \\
\text { Temperature Sensor }\end{array}$ & 0.4 & 1 & 6 & 2.4 \\
Servo Motor & 0.05 & 1 & & \\
& 4.5 & 1 & 6 & 0.15 \\
& & & 6 & 27 \\
\hline
\end{tabular}

Table 2 is the calculation of a smart fish feeder using solar energy. There are three types of sizing that are important to build this project which are solar panel sizing, solar charge controller and battery sizing. 
Table 2 - Calculation of the smart fish feeder using solar energy

\begin{tabular}{|c|c|}
\hline Component & Sizing calculation \\
\hline Solar Panel Sizing & $\begin{array}{l}\text { - Total Power Consumption per day }=29.5 \mathrm{Wh} / \text { day } \\
\text { - Solar Size in } \mathrm{W}=\text { Total load per Day/ Sun Hour per day } \\
\times \text { Derate Factor }\end{array}$ \\
\hline & $\begin{array}{l}=29.5 / 6 \times 0.77 \\
=3.78 \mathrm{~W}\end{array}$ \\
\hline
\end{tabular}

- According to NREL, typical overall derate factor is 0.77

- $\quad$ The average peak sun hour in Malaysia is around 4-6 Hours.

- $\quad$ Number of PV panels $=3.78 / 10=0.37=1$ module

- The system should be powered 1 module of $12 \mathrm{~V} 10 \mathrm{~W}$ Solar panel.

Solar Charge Controller
- $\quad$ PV Module Specification

$$
\begin{aligned}
& \mathrm{Pm}=10 \mathrm{Wp} \\
& \mathrm{Vm}=18 \mathrm{Vdc} \\
& \mathrm{Im}=0.58 \mathrm{~A} \\
& \mathrm{Voc}=21.5 \mathrm{~A} \\
& \mathrm{Isc}=0.62 \mathrm{~A}
\end{aligned}
$$

- $\quad$ Solar charge controller rating $=\mathrm{Isc} \times 125 \% \times 125 \%=$ $0.96 \mathrm{~A}$

- The array short circuit current (Isc) should be used, along with a 125 percent safety factor for cloud enhancement and another 125 percent safety factor for design margin, according to NEC.

- So the solar charge controller should be rated $10 \mathrm{~A}$ at $12 \mathrm{~V}$ or greater.

Battery Sizing

- $\quad$ Total appliances use $=29.5 \mathrm{Wh} /$ day

Nominal battery voltage $=12 \mathrm{~V}$

Days of Autonomy = 3 Days

- $\quad$ Battery Capacity $(\mathrm{Ah})=$ Total Watt-hours used by appliances per day $\times$ by Days of Autonomy/ $(0.85 \times$ $0.6 \times$ nominal battery voltage $)=29.5 \times 3 /(0.85 \times 0.6$ $\times 12)=14.46 \mathrm{Ah}$

- $\quad$ Based on that, the battery should be rated $12 \mathrm{~V} \mathbf{1 4 . 4 6}$ Ah for 3 day autonomy 


\subsection{Software Development}

Software development is an important part of every project especially due to the fact it uses to make a decision for the system by inserting the coding or instruction into the microcontroller. At this phase, Arduino IDE was as ESP8266 NodeMCU microcontroller. This software is specifically used to make and upload the coding sketch into ESP8266 NodeMCU. For this software, language use is Arduino language which a set of $\mathrm{C} / \mathrm{C}++$ functions that can be called from the code. The sketch undergoes minor changes (e.g. automatic generation of function prototypes) and then is passed directly to a $\mathrm{C} / \mathrm{C}++$ compiler. Fig. 3 shows the part of coding that is required to upload in NodeMCU. The servo motor will be connected to the pin $3 \mathrm{~V}$ power source, pin Ground and pin D7. In the coding the angle must be set to 90 degrees to rotate the servo motor. After that, the temperature sensor must be set in Pin D4 with the same power source and ground. The Blynk part in the coding will show the value of the temperature sensor continuously and the timer for the fish feeder also will show in the Blynk.

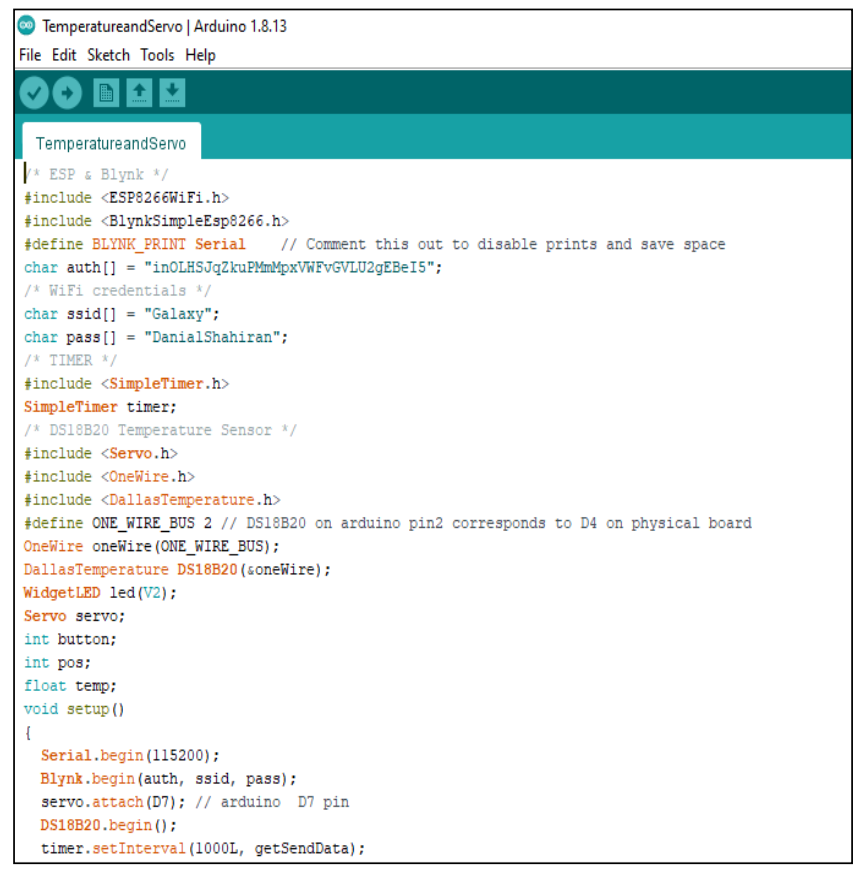

(a)

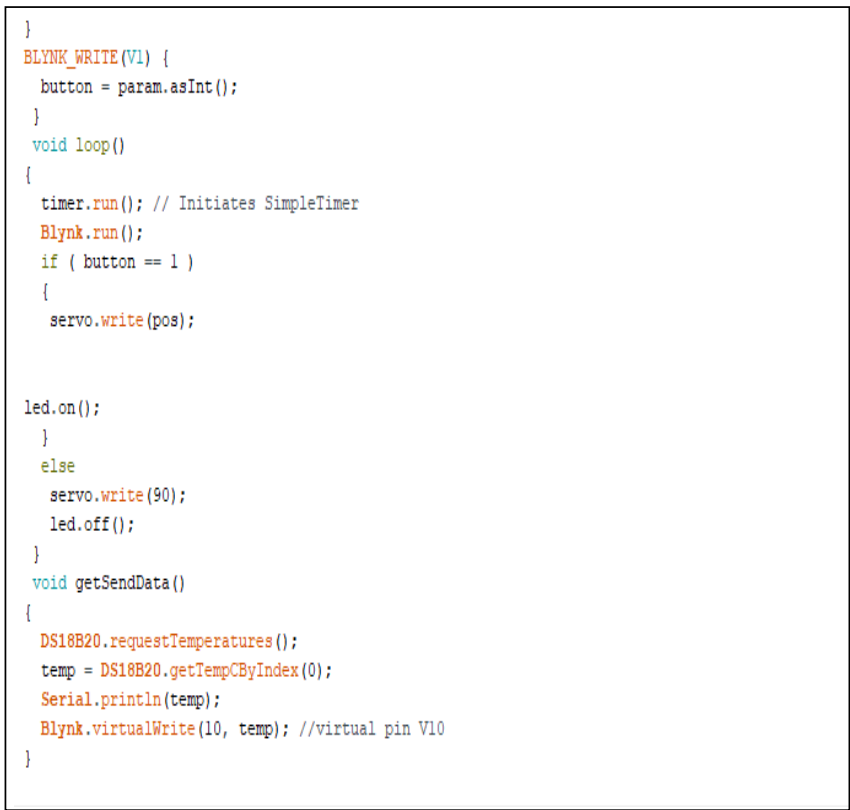

(b)

Fig. 3 - Coding in Arduino IDE; (a) first part of coding; (b) continuation of first part 
For this project, the button is set as "MANUAL FEED". The connected Port is a Virtual pin (V1) port which mention in the coding to connect to and control the servo motor. The angle is set as a variable depends on the preference. The reason it starts at a $90^{\circ}$ angle is due to the installation method and the rotation which is clockwise direction. If it starts with $0^{\circ}$, the motor will have stuck and cannot rotate because there is an obstacle. The mode used is a switch that will turn on and change the servo angle from $90^{\circ}$ to $40^{\circ}$ (depends on the angle set). When the button was push again, the servo will change from $40^{\circ}$ to $90^{\circ}$. The label when the button is on and off is set based on the servo angle set above. The font size is medium and the colour is green. The timer can set the label for the timer, connected port, angle output, start and stop the timer, font size and text colour. The label is set as "FEEDING TIMER 1" and "FEEDING TIMER 2". The port is connected to the V1 port. The start and stop timer depend on the time to feed the fish. The timer interval is in sec depends on the fish feed needed.

Fig. 4 shows the main interface for the Blynk application. The main feature is a timer, button and temperature. The button is set to manually feed the fish. If the feeding is not enough for fish the manual button will be pushed to give more pellets. The timer is automatically fed the fish every day by the time setting. The reason there is only two buttons because it is proven by previous research [11], two times a day already enough for maximal growth. The button can set the name for the button, connected port, angle output, mode, ON and OFF label, Font size, and text colour.

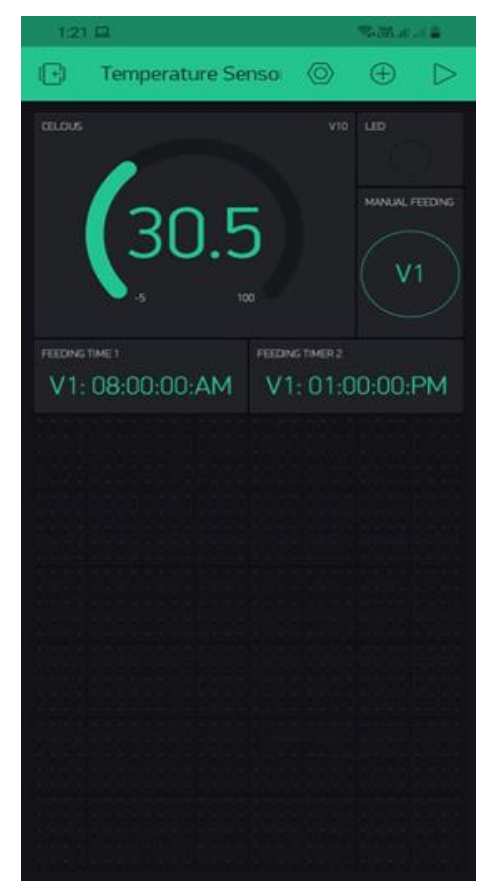

Fig. 4 - Blynk app design interfere

\section{Results and Discussion}

\subsection{System Performance}

The smart fish feeder system is powered by a $12 \mathrm{~V}$ battery with a solar panel to charge the battery. It also comes with NodeMCU. The whole system for the smart fish feeder system is built. For the supply part, solar energy is used while for the sensor part, the temperature sensor is used. The NodeMCU will receive the data from the sensors and also receive the command given to turn the servo motor from the Blynk app. The functionality test of the developed prototype work has been carried out on real scenarios and the overall system works well. Fig. 5 shows all the connections of the hardware component on this project. 


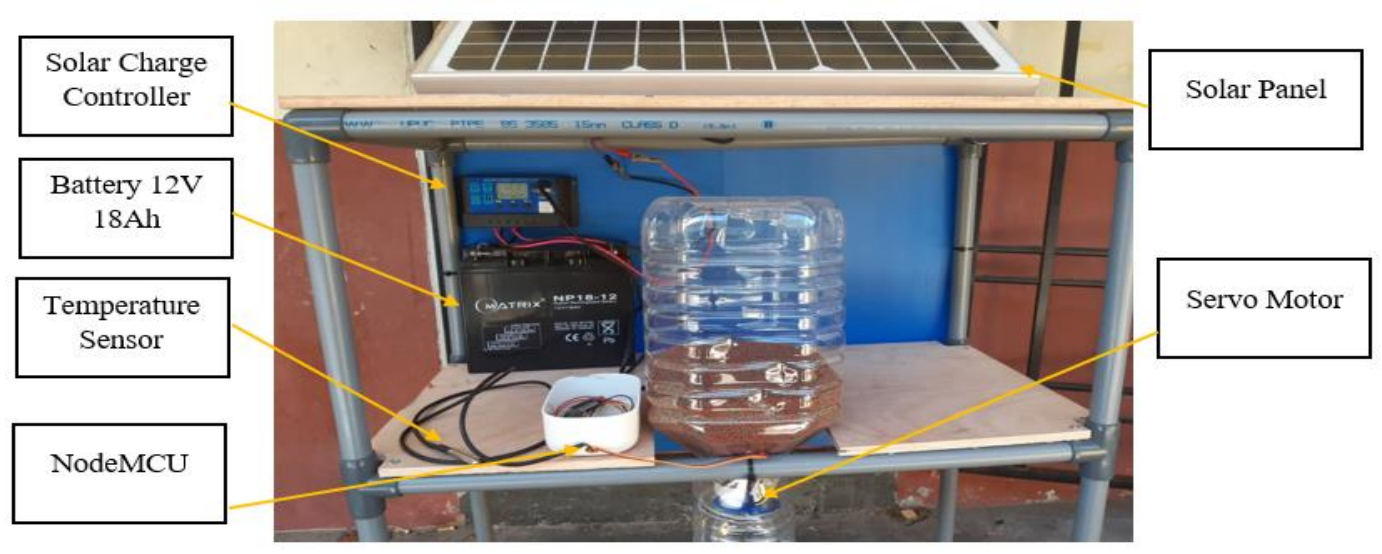

Fig. 5 - Connection of the hardware component

\subsection{Testing and Result Analysis with Blynk Apps}

There are three widgets used in the Blynk application which are the virtual button, virtual gauge and virtual timer, when the Blynk application is connected with NodeMCU. Fig. 6 shown that the virtual button, virtual gauge and virtual timer in the Blynk application when online. The Blynk application must be connected to the same Wi-Fi with NodeMCU. Figure 6 shows the Blynk and the NodeMCU are connected via Wi-Fi. After being connected to the Wi-Fi, the servo motor and temperature sensor are ready to function by using the Blynk application. Thus, the monitoring system Blynk interface is shown in Figure 6. The readings of the sensors are keep updated with the value of water temperature. Figure 6 demonstrates the framework created for automated mobile phone control of the project. The interface was combined into one main tab before running the test. By used Graphical Users Interface (GUI) in this Blynk system, users can monitor the real-time value for temperature sensor first before running the servo motor. One set of buttons were used to control the ON/OFF Servo motor. Basically, the user can control the servo motor and also monitor the real-time value of the temperature sensor which means the signal from NodeMCU given to Blynk and Feeding Timer 1 and Feeding Timer 2 operate accordingly to the input gives from the Blynk application.

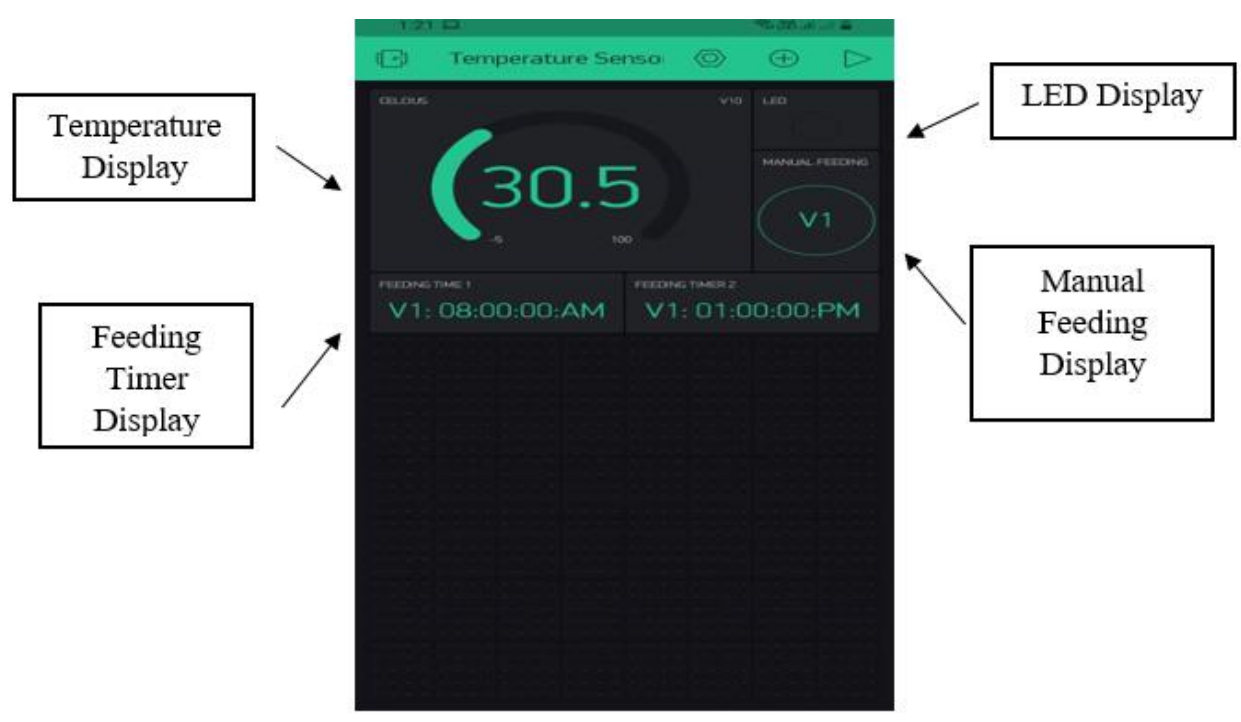

Fig. 6 - Blynk interface of monitoring system

\subsection{Smart Fish Feeder Operation Using Blynk Apps}

The operation of a fish feeder starts when Feeding Timer 1 and Feeding Timer 2 are set at a certain time in Blynk Apps. Figure 7 (a) below shows the operation of the fish feeder in this project. In this system, the servo motor will operate when the user sets a certain time to make a pellet drop in the fishpond. Besides, the user also can manually push the button to make sure all the fish get the same amount of pellet in Figure 7 (b) shows that the user touches a manual button to feeding the fish. At the screen, the user can get real-time data on water temperature. 


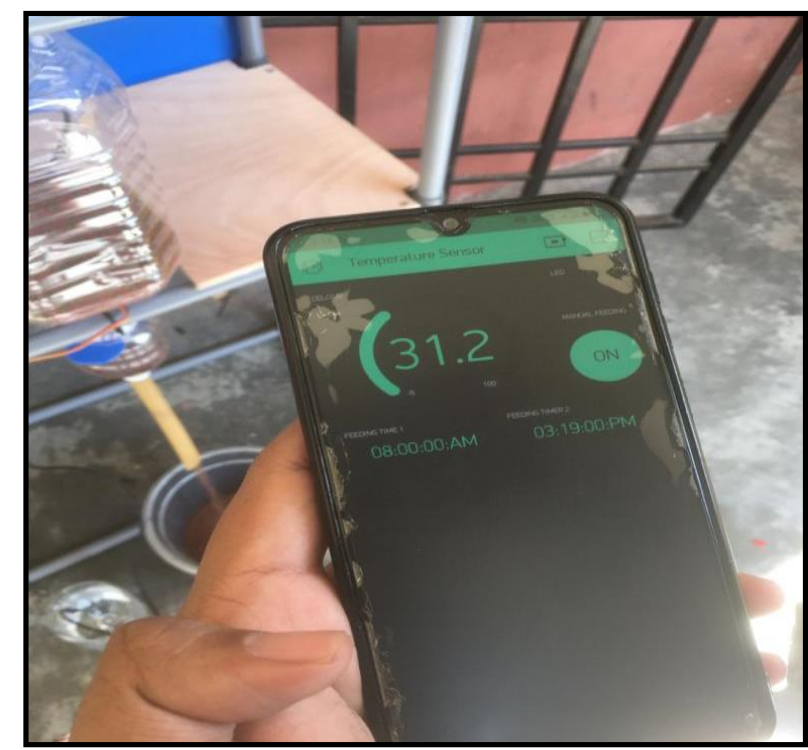

(a)

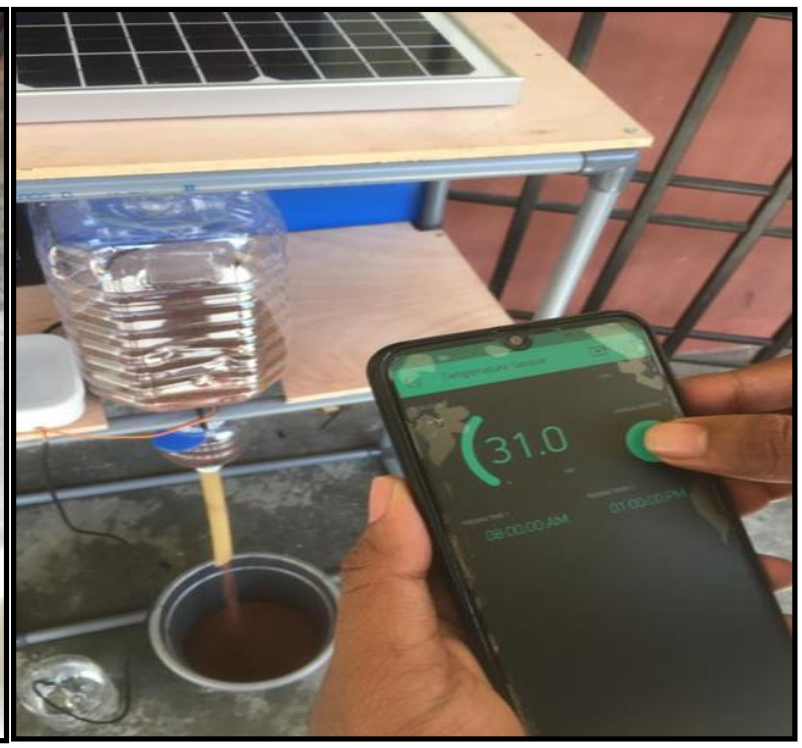

(b)

Fig. 7 - (a) fish feeder using timer; (b) fish feeder using manual button

\subsection{Result Analysis of Solar Panel Charge the Batteries}

The test was used to evaluate the solar panel's output voltage and the time taken to charging $12 \mathrm{~V}$ batteries. Solar charge controller and optical multimeter were used to calculate the output voltage of the solar. The research took place on 20 June 2021 and solar panels generated data from 10:30 a.m. until 3.00 p.m. Table 3 subsequently shows the reading of voltage reading during the charging of the battery by the solar panel.

Table 3 - Voltage during charging of the battery by solar panel

\begin{tabular}{cc}
\hline Time & Voltage(V) \\
\hline 10.30 a.m. & 12.1 \\
11.00 a.m. & 12.3 \\
11.30 a.m. & 12.4 \\
12.00 p.m. & 12.5 \\
12.30 p.m. & 12.6 \\
1.00 p.m. & 12.7 \\
1.30 p.m. & 12.8 \\
2.00 p.m. & 12.9 \\
2.30 p.m. & 12.8 \\
3.00 p.m. & 12.7
\end{tabular}

Figure 8 shows the results from the solar panel to charge the battery and the voltage reading is rises at peak hours around 10:30 a.m. to 3.00 p.m. This is because, at this hour, the sunlight produces the highest solar irradiance. 


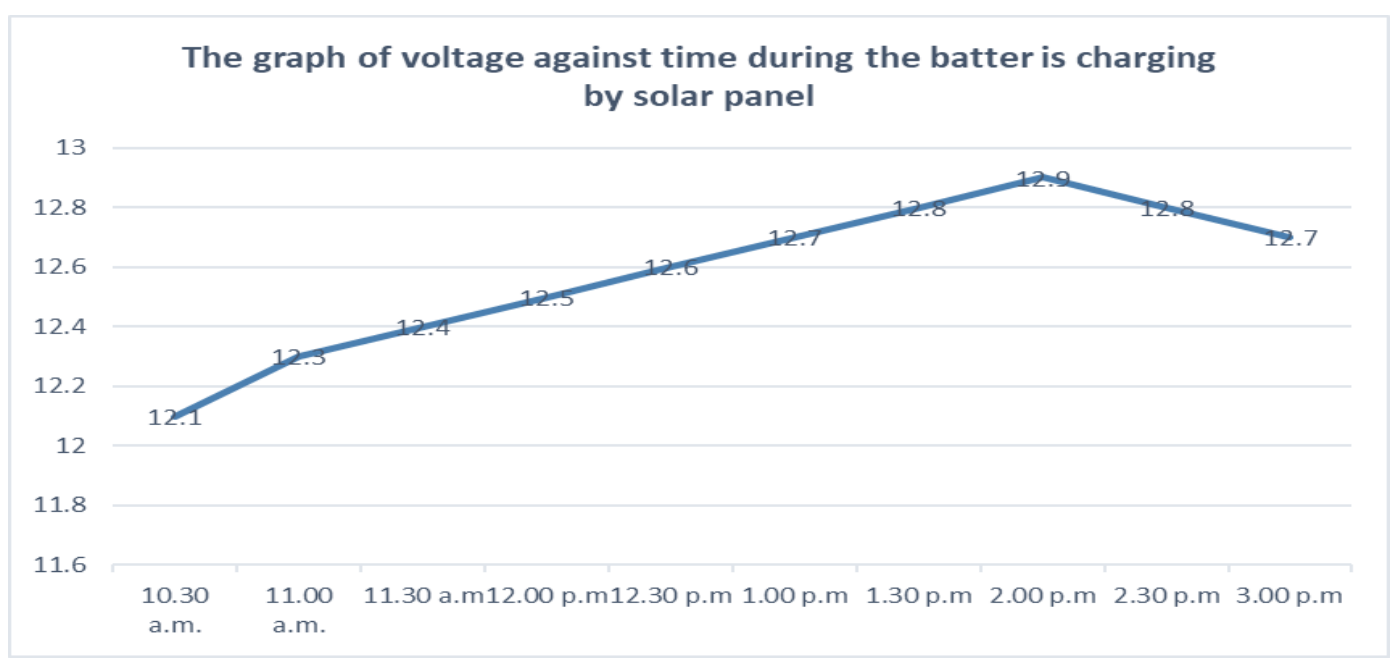

Fig. 8 - Charging voltage against the time of a day

\section{Conclusion}

In conclusion, the prototype of Smart Fish Feeder Using Solar Energy has been successfully developed and validated. All of the objectives have been achieved. From the results, Smart Fish Feeder Using Solar Energy can be operated during day and night. The Smart Fish Feeder Using Solar Energy is provided a simple mobile app connection platform, Blynk that can control the operation of the servo motor and manage to monitor the status of the temperature sensors. With the mobile app application, the user can operate the Smart Fish Feeder Using Solar Energy even not at home. Solar power supplies enough energy to drive the system and the battery to ensure the system is can be operated overnight. The results revealed that Smart Fish Feeder Using Solar Energy was focused on supporting small farmers and users to reducing their workload, help to reduce the time limit and minimize energy usage. The basic concept proved in this project will be served as fundamental evidence of a large-scale smart agriculture system taking into consideration the number of sensors and servo motor which may rescale the type of hardware design.

\section{Acknowledgment}

The authors would like to thank Faculty of Electrical and Electronic Engineering, Universiti Tun Hussein Onn Malaysia (UTHM) for supporting this project until completion.

\section{References}

[1] M. Lamnadi, M. Trihi, and A. Boulezhar, (2016). Study of a hybrid renewable energy system for a rural school in Tagzirt, Morocco, Proc. 2016 Int. Renew. Sustain. Energy Conf. IRSEC 2016, pp. 381-386, 2017

[2] Department of Mechanical Engineering, University of Malaya, Faculty of Engineering 50603 Kuala Lumpur, Malaysia (2011) A review on global solar energy policy

[3] Dept. of Statistics, M. (2019). Department of Statistics Malaysia Press Release. Department of Statistics Malaysia.

[4] Saikia, S. K. (2015). Food and Feeding of Fishes. What Do We Need to Know? Transylvanian Review of Systematical and Ecological Research, 17(1), pp.71-84

[5] V. K. Beena and K. Moinuddin, "Water Quality Measurement and Control from Remote Station for pisiculture using NI myRIO (2015). International journal for research in Electronic and Telecommunication, vol. 2, no. 4, pp. $16-21$

[6] A. Abdulrazaq, D. Mustapha, A. Ismail, M. Momoh, S. Abdulhamid, J. Obari, and M. Saliu (2018). LabVIEW Interfaced PC-Based Fishpond Monitoring System, ATBU Journal of Science, Technology and Education, vol. 6, no. 2, pp. 217-225

[7] N. A. J. Salih, I. J. Hasan, and N. I. Abdulkhaleq (2019). Design and implementation of a smart monitoring system for water quality of fish farms, Indonesian Journal of Electrical Engineering and Computer Science, vol. 14, no. 1, pp. $44-50$

[8] J. S. Diana, J. P. Szyper, T. R. Batterson, C. E. Boyd, and R. H. Piedrahita (1997). Water quality in ponds," Dynamics of pond aquaculture, pp. 53-72.

[9] N. Ezeanya, G. Chukwuma, K. Nwaigwe, and C. Egwuonwu (2015). Standard Water Quality Requirements and Management Strategies for Fish Farming (A Case Study of Otamiri River)," International Journal of Research in Engineering and Technology, vol. 4, no. 3, pp. 1-5 
[10] C. Z. Myint, L. Gopal, and Y. L. Aung, "Reconfigurable smart water quality monitoring system in IoT environment (2017), 2017 IEEE/ACIS 16th International Conference on Computer and Information Science (ICIS), pp. 435-440,

[11] Abdul Malik, Dr \& Abbas, Ghulam \& Kalhoro, Illahi \& Sajjad, Syed \& Shah, A \& Kalhoro, Hameeda \& Hafeezur-Rehman, Muhammad \& Ghaffar, Abdul. (2016). Effect of Feeding Frequency on Growth Performance, Feed Utilization and Body Composition of Juvenile Nile Tilapia, Oreochromis niloticus (L.) Reared in Low Salinity Water. Pakistan Journal of Zoology. 48. pp.171-177 